\title{
SEGURANÇA DO PACIENTE EM CENTRO CIRÚRGICO: PERCEPÇÃO DOS PROFISSIONAIS DE ENFERMAGEM
}

\author{
Patient safety in the surgical center: nursing professionals perceptions
}

\section{Seguridad del paciente en centro quirúrgico: percepción de los profesionales de enfermería}

\author{
Aline Tamiris Gonçalves Souza' (D), Tais Kele de Paula da Silva² (D), Aline Natalia Domingues ${ }^{3}$ (D), Silvia Helena Tognoli ${ }^{3}$ (D), \\ Aline Helena Appoloni Eduardo $0^{4}$, Juice Ishie Macedo 5 (D), Adriana Aparecida Mendes ${ }^{4 *}$
}

RESUMO: Objetivo: Conhecer as ações realizadas pelos profissionais de enfermagem direcionadas à segurança do paciente no ambiente de centro cirúrgico (CC), segundo discurso desses profissionais. Método: Trata-se de uma pesquisa de campo, de caráter exploratório e descritivo, com abordagem qualitativa. Os dados foram coletados em um CC por meio de entrevista gravada norteada por roteiro estruturado com quatro questões abertas fundamentadas no método do discurso do sujeito coletivo (DSC), com foco na prática da assistência segura para o paciente no CC. A amostra foi composta de 12 profissionais de enfermagem, sendo um auxiliar e 11 técnicos. Os dados foram organizados e analisados segundo método do DSC. Resultados: As respostas deram origem a seis discursos, que revelaram preocupação em manter a segurança do paciente por meio de identificação, comunicação entre equipe multiprofissional e paciente, prevenção de quedas, ações para a prática segura, comunicação intersetorial e manutenção de equipamentos. Conclusão: Os profissionais de enfermagem compreendem a importância da segurança do paciente no CC e consideram que o conjunto de práticas realizadas deve estar alinhado, de modo que minimize eventos adversos e proporcione assistência qualificada, em benefício da qualidade de vida do paciente. Palavras-chave: Segurança do paciente. Centros cirúrgicos. Enfermagem.

ABSTRACT: Objective: To know the actions aimed at patient safety performed by nursing professionals in Surgical Centers (SC), and in accordance with these professionals' perceptions. Method: This is an exploratory and descriptive field research with a qualitative approach. Data were collected in a SC through a recorded interview guided by a structured script with four open questions based on the Discourse of the Collective Subject (DCS) method, focusing on patient safe care practices in the SC. The sample was constituted by 12 nursing professionals, one of which was an auxiliary and 11 were technicians. Data were organized and analyzed according to the DCS method. Results: The answers resulted in six discourses that revealed concern with patient safety regarding identification, communication between multiprofessional team and patient, fall prevention, safe practices, intersectoral communication, and equipment maintenance. Conclusion: Nursing professionals understand the importance of patient safety in the SC, and consider that the set of practices performed should be aligned in a way that will minimize adverse events and provide qualified assistance in the benefit of the patient's quality of life. Keywords: Patient safety. Surgicenters. Nursing.

RESUMEN: Objetivo: Conocer las acciones tomadas por los profesionales de enfermería para la seguridad del paciente en el entorno del Centro Quirúrgico (CQ), según el discurso de estos profesionales. Método: Esta es una investigación de campo exploratoria y descriptiva, con un enfoque cualitativo. Los datos se recopilaron en un CQ a través de una entrevista grabada guiada por un guión estructurado con cuatro preguntas abiertas basadas en el método del Discurso del Sujeto Colectivo (CSD), con un enfoque en la práctica de la atención segura de los pacientes en el CQ. La muestra estuvo compuesta por

'Enfermeira pela Universidade de Araraquara. Enfermeira da Unimed de Araraquara, Hospital São Francisco - Araraquara (SP), Brasil.

2Enfermeira pela Universidade de Araraquara. Enfermeira da Unimed de Araraquara, Hospital Estadual de Américo Brasiliense - Américo Brasiliense (SP), Brasil. Doutoranda pela Escola de Enfermagem de Ribeirão Preto da Universidade de São Paulo (EERP/USP) - Ribeirão Preto (SP), Brasil.

Doutora em Ciência pela EERP/USP - Ribeirão Preto (SP), Brasil.

Doutora em Enfermagem pela Universidade Federal de Minas Gerais - Belo Horizonte (MG), Brasil.

*Autora correspondente: adrianaapmendes@yahoo.com.br

Recebido: 30/12/2019 - Aprovado: 02/04/2020

DOI: $10.5327 / Z 1414-4425202000020003$ 
12 profesionales de enfermería, un asistente y 11 técnicos. Los datos fueron organizados y analizados de acuerdo con el método DSC. Resultados: Las respuestas dieron lugar a seis discursos, que revelaron preocupación por mantener la seguridad del paciente a través de la identificación, la comunicación entre el equipo multidisciplinario y el paciente, la prevención de caídas, las acciones para la práctica segura, la comunicación intersectorial y el mantenimiento del equipo. Conclusión: Los profesionales de enfermería entienden la importancia de la seguridad del paciente en el CQ y consideran que el conjunto de prácticas realizadas debe estar alineado, para minimizar los eventos adversos y proporcionar asistencia calificada, en beneficio de la calidad de vida del paciente.

Palabras clave: Seguridad del paciente. Centros quirúrgicos. Enfermería.

\section{INTRODUÇÃO}

A cirurgia segura corresponde ao Segundo Desafio Global para a Segurança do Paciente, campanha proposta pela Organização Mundial da Saúde (OMS) com os objetivos de promover a melhoria da segurança cirúrgica e reduzir os índices de mortes e complicações durante a cirurgia ${ }^{1,2}$.

O conceito de cirurgia segura envolve medidas adotadas para redução do risco de eventos adversos que podem acontecer antes, durante e depois da cirurgia, tais como: prevenção de infecção de sítio cirúrgico (ISC), anestesia segura, equipe cirúrgica preparada e mensuração da assistência cirúrgica² ${ }^{2}$.

Ante essa necessidade, a OMS desenvolveu a Lista de Verificação de Segurança Cirúrgica (checklist cirúrgico), que deverá ser seguida pela equipe de profissionais da saúde, com o objetivo de minimizar erros considerados evitáveis que colocam em risco a vida e o bem-estar dos pacientes cirúrgicos ${ }^{2,3}$.

Assim, a implementação da Lista de Verificação de Segurança Cirúrgica deve ocorrer em três momentos distintos: no período que antecede a indução anestésica (identificação - sign in), antes da incisão cirúrgica (confirmação - timeout) e após o procedimento cirúrgico, ainda com o paciente em sala operatória (SO) (registro - sign out $)^{2}$.

Segundo a OMS, essa tarefa poderá ser realizada por membro da equipe de enfermagem da SO ou médico participante do procedimento cirúrgico ${ }^{4}$.

Entre as possibilidades de ocorrências de eventos adversos no ambiente hospitalar, destacam-se as ISC, que podem estar relacionadas à própria patologia ou aos procedimentos invasivos realizados, sendo responsável por gerar aumento na taxa de mortalidade e custos, assim como reinternação 5 . Pontua-se que em setores críticos, como é caso do centro cirúrgico (CC), ocorre parcela expressiva das infecções, pois a ferida operatória pode ser porta de entrada para microrganismos ${ }^{6}$.

Nesse contexto, a Associação Brasileira de Enfermeiros de Centro Cirúrgico, Recuperação Anestésica e Centro de
Material e Esterilização (SOBECC) descreve que a atuação da equipe no CC excede a capacidade técnica e de interação com o paciente e sua família ${ }^{7}$.

Dados de um estudo realizado em dois hospitais do Rio Grande do Norte revelam que a baixa adesão à Lista de Verificação de Segurança Cirúrgica possivelmente teve reflexo sobre a ocorrência de eventos adversos em cirurgia, tais como aumento da permanência no ambiente hospitalar, risco de reinternação, necessidade de terapia intensiva, mortalidade, entre outras situações ${ }^{8}$.

Destaca-se que no CC há número representativo de profissionais de enfermagem atuantes na assistência contínua, desempenhando papel importante no processo de segurança do paciente cirúrgico.

\section{OBJETIVO}

Conhecer as ações realizadas pelos profissionais de enfermagem direcionadas para a segurança do paciente no ambiente de um CC, segundo discurso desses profissionais.

\section{MÉTODO}

Trata-se de uma pesquisa de campo, de caráter exploratório e descritivo e de abordagem qualitativa, realizada em um CC de um hospital filantrópico no interior do estado de São Paulo/Brasil.

Foram convidados para participar do estudo os 35 profissionais de enfermagem (enfermeiros, auxiliares e técnicos de enfermagem) atuantes no CC segundo escala de serviço, sendo critérios de inclusão: profissional que atua no setor por período igual ou superior a 12 meses, considerado tempo necessário para adaptação às suas rotinas, e que aceitaram participar da pesquisa, com assinatura do termo de consentimento livre e esclarecido. Excluíram-se os ausentes no momento da coleta dos dados, por motivo de afastamento, férias ou folga. 
Entre os 35 profissionais, 23 não atenderam aos critérios de inclusão, totalizando em 12 o número de participantes, sendo um $(8,3 \%)$ auxiliar de enfermagem e $11(91,7 \%)$ técnicos de enfermagem, predominando o gênero feminino (9/75,0\%), em comparação ao masculino (3/25,0\%).

Quanto à idade, houve variação de 21 a 51 anos, com predominância $(8 / 66,7 \%)$ entre 21 e 30 anos. No que se refere à formação, nove (75\%) são técnicos de enfermagem formados de 1 a 10 anos, dois $(16,7 \%)$ com nível superior há 10 anos e um $(8,3 \%)$ auxiliar de enfermagem com tempo de formação superior a 10 anos.

Referente à função no setor, 83,3\% (10) são circulantes e $16,7 \%$ (2) agentes de transporte. Quanto ao tempo na função, 41,7\% (5) atuavam entre um e dois anos, 25\% (3) de três a quatro anos e $33,3 \%$ (4) por tempo superior a cinco anos.

As pesquisadoras realizaram as entrevistas nos períodos de manhã, tarde e noite, utilizando-se a escala de trabalho vigente no setor como referência para identificar e convidar os profissionais para participarem do estudo.

A coleta dos dados ocorreu em local reservado no CC, previamente indicado pelo enfermeiro responsável do setor. $O$ instrumento utilizado para a coleta dos dados foi composto de itens destinados à caracterização do participante e um roteiro de entrevista estruturado, elaborado com quatro questões norteadoras abertas, segundo a proposta do método do discurso do sujeito coletivo (DSC) ${ }^{9}$ :

1. Você sabe que a prática da assistência segura nas instituições de saúde está na agenda das prioridades, pois reflete diretamente na qualidade do cuidado prestado ao paciente. Você poderia falar sobre a segurança do paciente?;

2. Você tem conhecimento de que a equipe de enfermagem, continuamente durante os cuidados prestados ao paciente, realiza ações direcionadas à promoção da segurança do paciente no ambiente do CC? Você poderia falar sobre essas ações?;

3. A prática da assistência segura implementada pela equipe de enfermagem tem a finalidade de promover a segurança do paciente. Conte-me sobre a importância dessas ações no ambiente do CC;

4. Você considera que em seu cotidiano laboral no CC há a possibilidade de desenvolver novas ações direcionadas à segurança do paciente? Você poderia falar um pouco sobre isso?

As respostas obtidas foram registradas em gravador de voz e transcritas na íntegra após escuta exaustiva. Analisaram-se os dados segundo o método do DSC, que orienta a selecionar nas transcrições das entrevistas as figuras metodológicas definidas como expressões-chave $(\mathrm{ECH})$ e ideias centrais (IC) para a construção do DSC, fundamentadas na teoria das representações sociais ${ }^{9}$.

Atenderam-se aos aspectos éticos da pesquisa conforme determinações da Resolução n ${ }^{\circ}$ 466/2012, do Conselho Nacional de Saúde ${ }^{10}$. Esta pesquisa foi avaliada e aprovada pelo Comitê de Ética em Pesquisa, sob parecer n ${ }^{\circ} 1.768 .388$ e Certificado de Apresentação para Apreciação Ética (CAAE): 59561516.9.0000.5383.

\section{RESULTADOS}

Os resultados obtidos nas entrevistas por meio das quatro questões norteadoras foram analisados, resultando nos seis discursos apresentados a seguir.

\section{DSC 1: Preocupação com a identificação e a checagem de dados do paciente no centro cirúrgico}

Os profissionais de enfermagem afirmaram que, entre as práticas assistenciais, a identificação correta do paciente é fator primordial para assistência segura no CC.

Para mim, a segurança do paciente aqui dentro do CC é verificar a pulseira [...], o paciente chega [...] pergunta para ele o nome dele [...], a identificação antes de ele entrar. Perguntar como ele chama é uma segurança que você tem, o prontuário, data de nascimento, idade [...], o nome do médico, se é o cirurgião correto, essas são algumas medidas que a gente tem de segurança. O nome que ele falar você vai usar como referência no papel, a cirurgia que ele vai fazer [...], você fala: o senhor vai operar a perna direita? Ele: não, [...] é a esquerda. Então ele sabe, a maioria deles tem essa ciência [...], você tem que estar muito preocupado, para não ter erro de cirurgia, $[\ldots]$ se toma medicação, se tem alergia a alguma medicação, [...] às vezes, eles não sabem dizer [...], pergunta se ele tem alguma doença, [...] diabetes, pressão alta, se já fez algum tipo de cirurgia fora aquela, [...] identificar se o paciente permanece de jejum, [...] sem próteses dentárias, $[\ldots]$ conferir prontuário. $\mathrm{O}$ anestesista reforça na sala de cirurgia para ter certeza. A maior 
prioridade aqui no CC, [...] uma assistência segura verificando o paciente correto, se atentar às queixas dele e tentar dar a melhor assistência para ele.

\section{DSC 2: Importância da comunicação verbal entre equipe multiprofissional e paciente no centro cirúrgico}

Outro apontamento realizado pelos participantes foi sobre a importância da comunicação verbal entre os membros que compõem a equipe de profissionais atuantes no $\mathrm{CC}$ com foco na segurança do paciente.

A segurança do paciente acho que é de extrema importância no CC, ocorrem [...] erros, às vezes, por causa de comunicação $[. .$.$] com o paciente e [. .$. com o médico, [quando] não sabe se o paciente tem alguma doença ou não, às vezes, o paciente é confuso, idoso [...], é muito importante essa segurança para evitar que aconteçam falhas tanto no procedimento quanto na assistência prestada.

\section{DSC 3: Segurança do paciente relacionada ao risco de queda}

Os profissionais de enfermagem também demonstraram preocupação em relação ao risco de queda dos pacientes no CC, pois estes permanecem ou na maca ou na mesa cirúrgica no setor.

Então a segurança é: [...] o paciente está em cima de uma maca, tem que estar com grades levantadas, $[\ldots .$.$] estar perto do paciente [\ldots]$ na mesa de cirurgia, porque pode acontecer do paciente cair [...], tem que prestar muita atenção, cuidar bem do paciente no CC.

\section{DSC 4: Ações para a prática da assistência segura no centro cirúrgico}

Outro apontamento dos participantes foi referente à relevância em manter prática de ações direcionadas à manutenção da assistência segura no CC, minimizando eventos adversos.

No CC, $[\ldots]$ o paciente vai passar pela cirurgia, tem que estar tudo correto. A segurança é tudo
[...], porque a gente tem que ter certeza que é o paciente correto, cirurgia correta. O paciente chega no CC muitas vezes até com o pré-anestésico, $[\ldots]$ assim a gente não consegue confirmar todos os dados, e você pode direcionar um paciente no que [...] vai fazer, [...] de qual médico, você tem que saber a identificação correta do paciente, $[\ldots]$ porque podem ser dois pacientes com mesmo nome, $[\ldots]$ e ele $[\ldots]$ com pré-anestésico não pode confirmar. Você tem que buscar outras informações, como [...] o mapa cirúrgico, diretamente com o cirurgião. A segurança é tudo, [...] porque com essas ações evita acontecer erros de cirurgia, $[. .$.$] de paciente, se real-$ mente o paciente tem alergia ou não de uma medicação [...], identificar os acessos [...], contagem de compressa durante a cirurgia é uma segurança. Então tudo isso faz com que acabe virando uma sequência [...], você faz o certo, o médico faz, o anestesista faz e aí vai virando uma roda onde tudo funciona, [...] e mesmo que, de repente, alguém falhe, a enfermagem acaba identificando e conseguindo evitar [...] alguma coisa com o paciente, e a gente tem que prevalecer a segurança do paciente, sempre perguntando para evitar que aconteça algum transtorno. [...] Como eu venho falando sobre a segurança do paciente, $[\ldots]$ quando está na maca ou num berço, tem que levantar as grades para que o paciente não venha a cair [...], porque o paciente acaba voltando da anestesia muito agitado, $[. .$. com grade levantada $[\ldots]$ o paciente não vai cair.

\section{DSC 5: Relevância da comunicação intersetorial com foco na segurança do paciente no centro cirúrgico}

Destaca-se, nos resultados obtidos, a preocupação em manter a comunicação multiprofissional efetiva entre os setores, em benefício da segurança do paciente.

Eu acho, $[\ldots]$ para melhorar, tem que ter uma troca de informação, [...] do CC com o setor [...] sempre estar passando plantão de um para o outro corretamente. E falta este tipo de informação, [...] seria internação, emergência, CC, tem que estar 
uma coisa ligada com a outra, [...] uma troca de informação, quando uma dessas áreas falha, a outra tem que dar conta de qualquer jeito para conseguir ir atrás [...], imagina no final da cirurgia você descobre que o paciente tem hepatite, HIV, tudo bem que você tem que fazer a proteção já primária [...], mas têm coisas que você não usa, de repente, óculos de proteção, alguma coisa você não vai usar ali na hora e, às vezes, o paciente tem $[. .$.$] tuberculose, pseudomonas,$ acineto, H1N1, por exemplo, ninguém fica com uma N95 todo dia aqui no CC, a gente só usa quando é necessário. Orientar o paciente sobre a cirurgia, $[\ldots]$ o que trazer de exames aqui para o CC, sobre tricotomia, se tem que ser feita ou não, sobre o uso de medicação em jejum ou não, porque a gente enfrenta bastante problema, tem paciente que não toma a medicação para pressão alta porque ele informa que não podia tomar a água, $[\ldots]$ mas tem que orientar melhor ele sobre isso. Muitas vezes o paciente tem alergia a alguma medicação, então [...] eu acho que a informação e a comunicação são muito importantes, deveria ser reforçada, $[. .$.$] tanto do prontuário quanto$ verbalmente, $[\ldots]$ essa ligação entre os setores, comunicar sempre a supervisão ou médico, e qualquer intercorrência.

\section{DSC 6: Importância da manutenção dos equipamentos para segurança física do paciente no centro cirúrgico}

Outro fato evidenciado pelos participantes foi a necessidade de manutenção dos equipamentos utilizados no CC, com a finalidade de manter a segurança física do paciente enquanto permanecer no setor.

Eu acho [...] que a única coisa que a gente podia ajudar a melhorar [...] é esse negócio [...] de quartos, $[\ldots]$ acho que o paciente devia pelo menos já subir com o quarto dele, [...] vai sair da cirurgia [...] se vai a tal quarto, ou quando não tem, às vezes, a gente segura o paciente a noite inteira aqui porque a gente fica com dó de mandar e ficar deitado numa maca [...] entendeu? [...] Às vezes, as macas não são adequadas, às vezes, você vai levando o paciente, a rodinha da maca rolando parece $[\ldots]$ um pula-pula, então acontece isso. Eu acho que melhoraria para o paciente, porque, às vezes, o paciente fez uma cirurgia e você vai com ele para o quarto, aquela maca começa a rodinha jogando, jogando, e eu acho que não é bom para o paciente, $[\ldots]$ as macas não têm uma segurança [...], aí vem você ergue a maca, a rodinha cai do pé da maca, então está o risco de causar um acidente no paciente, $[\ldots]$ nunca aconteceu comigo, mas pode acontecer, às vezes, nós [...] tirando daqui de dentro as macas vazias e acontece de a rodinha cair da maca. E se estivesse com paciente em cima? É perigoso, o risco de que pode causar um acidente com o paciente, então eu acho que tem que ter melhoria nessas coisas.

\section{DISCUSSÃO}

O ambiente hospitalar oferece diversos tipos de riscos à saúde dos pacientes, favorecendo o aumento da permanência no processo de recuperação. Portanto, é essencial o papel do profissional na identificação e na checagem de situações que podem comprometer a segurança do paciente, assim como a importância da avaliação e da implementação de medidas de prevenção à exposição aos riscos e aos prejuízos decorrentes da assistência ${ }^{11}$.

A Lista de Verificação de Segurança Cirúrgica tem a finalidade de garantir que ações básicas direcionadas ao paciente, em cumprimento às Metas Internacionais de Segurança do Paciente, sejam rotineiras, proporcionando melhorais no processo de comunicação e nas atividades desenvolvidas entre as equipes profissionais, no local onde a assistência seja prestada, independentemente das características da instituição de saúde ${ }^{2,3}$.

Neste estudo, de acordo com relato dos profissionais, houve destaque para a preocupação com a identificação correta do paciente no CC, como forma de minimizar eventos adversos e prejuízos à saúde do paciente.

Estudo realizado em Porto Alegre (RS) com a finalidade de conhecer sobre o uso de pulseira de identificação em pacientes internados revelou que informações como nome, número de registro e integridade foram contempladas. Os autores ainda destacam a importância da participação e da conscientização dos pacientes, da equipe e dos familiares 
visando ao cumprimento das práticas relacionadas à cultura de segurança do paciente ${ }^{12}$.

Outro estudo, realizado no Rio de Janeiro com o objetivo de analisar os procedimentos de identificação de pacientes críticos com foco na pulseira de identificação, apontou que 96\% dos pacientes investigados apresentavam pulseiras de identificação devidamente colocadas, fortalecendo a prática das ações propostas para segurança do paciente e a qualidade da assistência de enfermagem ${ }^{13}$.

Considera-se que a identificação correta do paciente corresponde à primeira ação das etapas que compõem o período perioperatório, sendo determinante para a garantia da segurança do paciente no CC.

Outro fato revelado na pesquisa, por meio das respostas obtidas, foi a preocupação dos participantes em relação ao processo de comunicação entre o paciente e a equipe multiprofissional, como forma de minimizar eventos adversos no processo da assistência.

Segundo as determinações descritas na Lista de Verificação de Segurança Cirúrgica, destaca-se o fortalecimento de práticas seguras associadas à comunicação efetiva entre os profissionais atuantes no setor ${ }^{2,3}$.

Estudo de revisão integrativa, realizado em bases de dados nacionais com a finalidade de analisar as publicações científicas que abordavam a atuação do enfermeiro com foco na promoção da segurança do paciente cirúrgico, revelou que, entre os 28 estudos analisados, ainda há ausência de diálogo entre paciente e profissionais sobre seus medos, ansiedades e dúvidas no pós-operatório ${ }^{4}$.

A comunicação efetiva do paciente com a equipe que vai realizar sua cirurgia é essencial, pois contribui para a identificação precoce de possíveis eventos adversos, minimizando ou eliminando suas ocorrências.

Outra situação revelada nas respostas obtidas neste estudo foi a preocupação com as quedas. Aponta-se que esse tipo de ocorrência pode gerar impactos negativos no processo de mobilidade dos pacientes, entre eles, o receio, a ansiedade e a depressão, predispondo ao aumento do risco para outras quedas ${ }^{14}$.

Dados da OMS apontam que a queda é responsável por 646 mil mortes mundialmente, com destaque para os idosos, sugerindo práticas como educação, organização de ambientes seguros e políticas direcionadas para reduzir esse tipo de ocorrência ${ }^{15}$.

Sabe-se que quedas de pacientes em áreas hospitalares estão relacionadas a fatores do ambiente físico, tais como desnivelamento em pisos, objetos caídos ao solo, mobílias com alturas inadequadas, idade superior a 85 anos, histórico recente de queda, redução da mobilidade, incontinência urinária, hipotensão postural e uso de medicamentos ${ }^{14}$.

Ressalta-se a importância da prevenção de quedas de pacientes no CC, pois, segundo relatos dos participantes deste estudo, aqueles se encontram sedados ou em situação de confusão mental relacionada aos medicamentos anestésicos, o que favorece o aumento do risco de queda, pois se encontram em macas, mesas operatórias ou leitos com espaço reduzido para acomodação e mobilidade.

É essencial a vigilância constante dos pacientes no CC, assim como a manutenção da rotina de grades de proteção elevadas como alternativa para reduzir o risco de quedas. Outra prática sugerida é manter a vigilância e a permanência ao lado do paciente até que ele esteja em condição segura.

No CC, realizam-se tarefas complexas que exigem dos profissionais atenção nos cuidados com o paciente e agilidade e precisão na execução das práticas assistenciais ${ }^{4}$. Mundialmente há preocupação com a qualidade da assistência relacionada aos procedimentos cirúrgicos e anestésicos em função do elevado número de eventos adversos ${ }^{3}$.

Em relação à atuação do enfermeiro na prática da segurança do paciente, estudo de revisão integrativa identificou, em artigos publicados no período de 2013 a 2017, que a implementação de protocolos como a sistematização da assistência de enfermagem perioperatória (SAEP) e a Lista de Verificação de Segurança Cirúrgica foram fundamentais para garantir a segurança do paciente, servindo como facilitadores para a identificação e a notificação de eventos adversos, principalmente pelos enfermeiros. Destacou-se, ainda, a relevância da capacitação como forma de qualificar a assistência e minimizar os eventos adversos ${ }^{16}$.

Nesse contexto, é possível afirmar que o enfermeiro exerce papel fundamental no direcionamento da equipe de enfermagem para o cumprimento da assistência segura com qualidade no perioperatório. Portanto se destaca a importância de capacitação e treinamento dos profissionais atuantes no CC, fortalecendo as práticas assistenciais seguras em benefício ao paciente.

Ressalta-se que a comunicação efetiva entre os membros da equipe cirúrgica proporciona benefícios diretos ao paciente, destacando-se a importância da comunicação entre os setores no momento de sua transferência como forma de segurança para o paciente e para a equipe que o assiste. As falhas na comunicação pela ausência de informações necessárias estão relacionadas entre as principais causas que contribuem para os eventos adversos ${ }^{17}$. 
A Lista de Verificação de Segurança Cirúrgica é essencial nesse processo de comunicação na rotina do CC, considerando a preconização das Metas Internacionais de Segurança do Paciente, a qual ressalta a necessidade de comunicação efetiva entre as equipes ${ }^{3}$.

Os resultados de estudo realizado em hospital filantrópico no município de São Paulo com o objetivo de conhecer a experiência de profissionais que construíram protocolo direcionado para a passagem de plantão entre o CC e a unidade de terapia intensiva revelaram que a comunicação entre profissionais é fundamental para o planejamento adequado das necessidades e para o segmento do cuidado ao paciente crítico com eficiência e segurança ${ }^{18}$.

Também vale ressaltar a importância do papel do enfermeiro em manter atualizadas e registradas informações sobre a manutenção preventiva dos equipamentos de transporte e acomodação de pacientes, com a finalidade de reduzir a ocorrência de quedas e outros eventos adversos.

Os resultados de estudo realizado com 220 enfermeiros de CC de diferentes regiões no Brasil com o intuito de descrever as recomendações segundo os enfermeiros para as boas práticas de segurança do paciente apontaram que recursos físicos, humanos e materiais adequados contribuem para a qualidade da assistência ${ }^{19}$. Portanto a inserção de rotinas direcionadas à avaliação e à manutenção de condições ideais do ambiente no CC é necessária para o adequado desempenho das práticas assistenciais voltadas à segurança do paciente.

\section{CONSIDERAÇÕES FINAIS}

Os resultados desta pesquisa revelaram que os profissionais de enfermagem compreendem a importância da segurança do paciente no CC, pois apontaram que, para essa finalidade, são fundamentais práticas assistenciais essenciais, tais como: identificação do paciente, conferência de prontuário, comunicação interpessoal entre a equipe multiprofissional e o paciente, checagem e manutenção de equipamentos, visando minimizar eventos adversos.

Portanto é necessário considerar que a segurança do paciente é mais abrangente do que apenas checagens, envolvendo um conjunto de práticas que deverão estar alinhadas para que os eventos adversos possam ser minimizados e a assistência seja qualificada de forma integral, em benefício da qualidade de vida do paciente.

Considera-se como limitação do estudo a ausência da participação de enfermeiros, pois seu papel gerencial no cuidado dos pacientes cirúrgicos, bem como dos profissionais de enfermagem atuantes no CC, contribui diretamente para as práticas assistenciais seguras para o paciente, fato que poderia fortalecer os resultados deste estudo.

Outro fato a ser considerado é a não inclusão de outros profissionais atuantes no CC, em função do tempo reduzido para a pesquisa. Sugere-se que estudos futuros possam ampliar essa temática, com número ainda limitado de publicações.

\section{REFERÊNCIAS}

1. Grabois V, Oliveira Júnior J. Cirurgia segura. In: Bopsin PS, Ribas EO, Silva DM, editores. Guia prático para segurança do paciente. Porto Alegre: Moriá; 2019.

2. Brasil. Ministério da Saúde. Segundo desafio global para a segurança do paciente: Cirurgias seguras salvam vidas [Internet]. Brasil: Ministério da Saúde; 2009 [acessado em 28 dez. 2019]. Disponível em: http://bvsms.saude.gov.br/bvs/publicacoes/ seguranca_paciente_cirurgia_salva_manual.pdf

3. Elias ACGP, Schmidt DRC, Yonekura CSI, Dias AO, Ursi ES, Silva RPJ, et al. Evaluation of the adherence to the safe surgery checklist at the public university hospital. Rev SOBECC. 2015;20(3):12833. http://doi.org/10.5327/Z1414-4425201500030002

4. Henriques AHB, Costa SS, Lacerda JS. Nursing care in surgical patient safety: an integrative review. Rev Cogitare Enferm. 2016;21(4):1-9. http://dx.doi.org/10.5380/ce.v21i4.45622
5. Fassini $P$, Hahh GV. Riscos à segurança do paciente em uma unidade de internação hospitalar: concepções da equipe de enfermagem. Rev Enferm UFSM. 2012;2(2):290-9. http://dx.doi.org/10.5902/217976924966

6. Ouriques $\mathrm{CM}$, Machado ME. Nursing in the process of sterilization of materials. Rev Texto Contexto Enferm. 2013;22(3):695-703. http://dx.doi.org/10.1590/S0104-07072013000300016

7. Campos JAR, Costa ACB, Dessotte CAM, Silveira RCCP. Scientific production in perioperative nursing from 2003 to 2013. Rev SOBECC. 2015;20(2):81-95. https://doi.org/10.5327/Z1414-4425201500020004

8. Freitas, MR, Antunes AG, Lopes BNA, Fernandes FC, Monte LC, Gama ZAS. Avaliação da adesão ao checklist de cirurgia segura da OMS em cirurgias urológicas e ginecológicas, em dois hospitais de ensino de Natal, Rio Grande do Norte, Brasil. Cad Saúde Pública. 2014;30(1):137-48. https://doi. org/10.1590/0102-311X00184612 
9. Lefevre F, Lefevre AMC. Pesquisa de representação social: um enfoque qualiquantitativo: a metodologia do discurso do sujeito coletivo. 2a ed. Brasília: Líber Livro; 2012.

10. Brasil. Conselho Nacional de Saúde. Resolução n 466 , de 12 de dezembro de 2012. Diretrizes e normas regulamentadoras de pesquisas envolvendo seres humanos [Internet]. Brasília; 2012 [acessado em 30 dez. 2019]. Disponível em: http://conselho. saude.gov.br/resolucoes/2012/Reso466.pdf

11. Oliveira Junior NJ. Segurança do paciente: o checklist da cirurgia segura em um centro cirúrgico ambulatorial [dissertação]. Porto Alegre: Universidade Federal do Rio Grande do Sul; 2015.

12. Hoffmeister LV, Moura GMSS. Uso de pulseiras de identificação em pacientes internados em um hospital universitário. Rev Latinoam Enferm. 2015;23(1):36-43. https://doi. org/10.1590/0104-1169.0144.2522

13. Macedo MCS, Almeida LF, Assad LG, Rocha RG, Ribeiro GSR, Pereira LMV. Patient identification through electronic wristband in an adult general intensive care unit. Rev Enferm Referência. 2017;4(13):63-70. https://doi.org/10.12707/RIV16087

14. Brasil. Ministério da Saúde. Protocolo Prevenção de Quedas [Internet]. Brasília: Ministério da Saúde; 2013 [acessado em 28 dez. 2019]. Disponível em: https://www20.anvisa. gov.br/segurancadopaciente/index.php/publicacoes/item/ prevencao-de-quedas

15. Who Health Organization (WHO). Global report on falls prevention in older age [Internet]. 2018 [acessado em $27 \mathrm{dez} .2019$ ]. Disponível em: https://www.who.int/news-room/fact-sheets/ detail/falls

16. Lopes TMR, Machado AVA, Silva AS, Santos TJX, Raiol IF, Miranda AS, et al. Nursing actions in patient safety at a surgical center: integrative literature review. REAS. 2019;(Supl. 26):1-10. https:// doi.org/10.25248/reas.e769.2019

17. Pancieri AP, Santos BP, Avila MAG, Braga EM. Safe surgery checklist: analysis of the safety and communication of teams from a teaching hospital. Rev Gaúcha Enferm. 2013;34(1):71-8. http://dx.doi.org/10.1590/S1983-14472013000100009

18. Sousa CS, Souza RCS, Gonçalves MC, Diniz TRZ, Cunha ALSM. Effective communication between surgical center and intensive care unit. Rev SOBECC. 2014;19(1):44-50. http://dx.doi.org/10.4322/ sobecc. 2014.004

19. Gutierres LS, Santos JLG, Peiter CC, Menegon FHA, Sebold LF, Erdmann AL. Good practices for patient safety in the operating room: nurses' recommendations. Rev Bras Enferm. 2018;71(Supl. 6):2775-82. http://dx.doi.org/10.1590/0034-7167-2018-0449 